\title{
Unilateral exophtalmos caused by an invasive giant prolactinoma
}

\begin{abstract}
We report the case of a 40-year-old male presenting with an unilateral exophtalmos due to a large tumoral mass invading the skull base. Magnetic resonance imaging (MRI) showed extension of a large tumour $(8 * 6 * 6 \mathrm{~cm})$ into the right orbit. Endocrine evaluation revealed elevated serum prolactin (PRL) levels $(161290 \mathrm{ng} / \mathrm{mL}, \mathrm{NR}<12.07)$ leading to the diagnosis of an invasive giant prolactinoma. Treatment with Bromocriptine resulted in rapid decrease of serum PRL ( 3 months after initiation of treatment), reduction of tumour size with improvement of the exophtalmos. With reference to this case, we review the features of invasive giant prolactinomas. We point out the use of Dopamine agonists in the treatment of prolactinomas with such atypical presentation.
\end{abstract}

Volume 4 Issue 3 - 2017

\author{
Najoua Lassoued, Faten HadjKacem, Nadia \\ Charfi and Mohamed Abid \\ Endocrinology department, Tunisia
}

Correspondence: Najoua Lassoued, Endocrinology department, CHU Hedi Chaker, Sfax, Tunisia, Tel +21694318468, Email najwalassoued@yahoo.fr

Received: May 14, 2016 | Published: April 03, 2017

Keywords: invasive, giant, prolactinoma, unilateral, exophtalmos

\section{Introduction}

Invasive giant prolactinoma is a large prolactinoma (over $4 \mathrm{~cm}$ in dimension) presenting with very high serum PRL concentrations (more than $1000 \mathrm{ng} / \mathrm{mL}$ ) and massive extension into the surrounding structures. ${ }_{1,2}$ Unlike microadenomas, these tumours often present with visual symptoms due to compression of the optic chiasm from upward growth. Orbital extension is very unusual and may lead to proptosis.

We report a patient with a giant prolactinoma revealed by an exophtalmos owing to an intraorbital extension and extensive skull base involvement.

\section{Case presentation}

A 40-year-old male complained of right proptosis, intermittent unilateral nasal obstruction and headaches that had persisted for 3 months. He had no previous history of trauma. He was admitted to the ENT department. Ophtalmological examination showed a reductible axial right-sided exophtalmos. The patient had no neurological deficits. The nasal endoscopy found a large submucosal bulging on the superior and posterior wall of the nasopharynx which infiltrates the posterior part of the nasal septum. Cerebral tomodensitometry showed a large tumoral mass of the skull base which invades the sellar and parasellar region, the chiasm region, the right middle cranial fossa and extends into the rear face of the petrous bone with osteolysis of the sphenoid. The nasopharynx biopsy found a reactive benigne lymphoid hyperplasia of the nasopharynx mucosa. MRI revealed a heterogenous pituitary giant adenoma of $84 * 68 * 64 \mathrm{~mm}$ in diameter, isointense to grey matter on $\mathrm{T} 1$, hyperintense on $\mathrm{T} 2$ and enhanced strongly after injection of Gadolinium. The tumour extended into the nasal cavity and the right orbit, invading the optic canal and the superior orbital fissure with a right exophtalmos grade III (Figure 1).

The patient was transferred to Endocrinology department. We found now the history of erectile dysfunction with gynecomastia on examination. There appeared to be no other signs of endocrine dysfunction. Thyroid function tests were normal as well as morning serum cortisal and basal serum growth hormone (GH). PRL levels were markedly elevated [161 $290 \mathrm{ng} / \mathrm{mL}$ (NR 0.5 12.07)] with low gonadotrophin (FSH $1.7 \mathrm{mUI} / \mathrm{mL}$; LH $1.64 \mathrm{mUI} / \mathrm{mL})$ and testosterone levels [2.01 ng/mL (NR 1.66_8.11)].

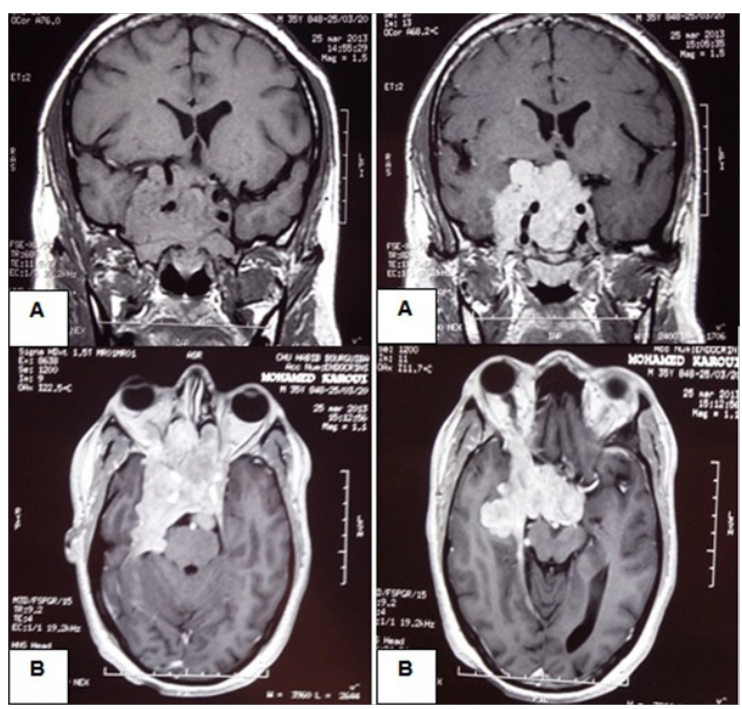

Figure I MRI of the pituitary region revealed a giant, invasive tumour with extension into nasal cavity and the right orbit with a right exophtalmos (A. coronal, and B. transverse sections).

Based on the serum PRL levels and the imaging appearances, the diagnosis of an invasive giant prolactinoma was made and the patient was started on treatment. BRC was initially administred at a dose of $1.25 \mathrm{mg} /$ day and progressively increased to a total dose of $7.5 \mathrm{mg} /$ day. Three months after bromocriptine therapy initiation, PRL levels were reduced to $41078 \mathrm{ng} / \mathrm{mL}$ with improvement of the exophtalmos. Follow-up imaging showed moderate shrinkage of the tumour $(66 * 42$ $\mathrm{mm})$.

\section{Discussion}

Giant prolactinomas represent only 2-3\% of all PRL secreting tumours. Their definition is restricted to pituitary adenomas with a large size (diameter $>40 \mathrm{~mm}$ ), very high PRL levels ( $>1000 \mathrm{ng} / \mathrm{mL}$ ) with no concomitant $\mathrm{GH}$ or ACTH secretion, ${ }^{3}$ and clinical symptoms 
induced by hyperprolactinemia or mass effect. They are much more frequent in man than in women with a male/female Ratio of $6.5 / 1{ }^{4}$

The most common endocrine symptoms are infertility, menstrual irregularity and galactorrhea in women and hypogonadism, decreased libido, infertility, erectile dysfunction and gynecomastia in men. ${ }^{5}$ These symptoms are often present but neglected for a long period of time and diagnosis is made only when neurologic complications occur due to the tumour invasive growth.

Atypical presentations for pituitary tumours are quite frequent depending on the extension into the surrounding intracranial structures.

Exophtalmos have been the subject of numerous case reports in the literature. ${ }^{6-8}$ Orbital invasion through the optic canal or orbital fissures is the most likely way in which exophtalmos may have developed. The exophtalmos may also be the result of inflammation and venous congestion in the orbit due to the tumour behind. ${ }^{9}$ Our patient presented with proptosis because of an orbital invasion which had occurred through the superior orbital fissure.

In very rare case, a large anterior tumoral extension into the nasopharynx has been described and can potentially produce nasal obstruction, epistaxis and respiratory problem. ${ }^{10-13}$ If endocrinological disturbances are not obvious, this may lead to inappropriate biopsy of the tumour ${ }^{12,14}$ which has occured in our case. A full pituitary hormonal evaluation is thus compulsory in the assessment of every large skull base tumour.

As in macroprolactinomas, Dopamine Agonist (DA) therapy is the primary treatment for giant prolactinomas as it has been proved to be effective in reducing PRL levels and inducing significant tumour shrinkage..$^{1,2,14,15}$ In contrast to the time required to reduce PRL levels and tumour size, the effect of DA on visual disorders is rapidely apparent within days after initiation of the treatment. ${ }^{2}$ There are several reports of rapid resolution under medical treatment alone of proptosis induced by giant prolactinomas. ${ }^{8,9,16}$ These effects were also observed with our patient who showed a remarkably good response to BRC with reduction of proptosis, reduction in PRL levels and moderate shrinkage of the tumour on a 3 month follow-up MRI.

Regarding the type of DA, even though there is no proof of a higher efficacy of cabergoline (CBG) over BRC in the management of giant prolactinomas, $\mathrm{CBG}$ is certainly better tolerated and generally recommended as first-line treatment. Furthermore, total surgical removal of giant prolactinomas that expand into different directions in the supra, para and infra sellar areas is very difficult or even impossible with a higher morbidity and mortality rates than for smaller, non invasive adenomas. Extensive neurosurgery should therefore be restricted to some acute complications or to treatment resistance.

There haven't been yet reliable predictors of tumour response in patient with giant prolactinomas. There is no correlation between age, gender, baseline PRL level, tumour size and the tumour response. A close vigilance must be therefore maintened to identify the need for surgical decompression.

\section{Conclusion}

Giant prolactinomas are rare tumours raising both diagnostic and therapeutic challenges. Atypical presentations are quite frequent and the diagnostic is made when neurologic complications occur rather than from long standing unrecognized endocrine symptoms. Pituitary hormonal evaluation is therefore mandatory in the assessment of every large skull base tumour.

Orbital extension by giant prolactinoma is an extremely unusual occurrence and the clinician needs to be wary of this presentation when the patient is complaining only of exophtalmos.

\section{Acknowledgements}

None.

\section{Conflicts of interest}

The author declares there is no conflict of interest.

\section{References}

1. Corsello SM, Ubertini G, Altomare M, et al. Giant prolactinomas in men: efficacy of cabergoline treatment. Clin Endocrinol (Oxf). 2003;58(5):662-670.

2. Shrivastava RK, Arginteanu MS, King WA, et al. Giant prolactinomas: clinical management and long-term follow up. $J$ Neurosurg. 2002;97(2):299-306

3. Dominique Maiter, Etienne Delgrange. The challenges in managing giant prolactinomas. Eur J Endocrinol. 2014 ;170(6):R213-R227.

4. Yu C, Wu Z, Gong J. Combined treatment of invasive giant prolactinomas. Pituitary. 2005;8(1):61-65.

5. Rahmanian M, Hamidreza AM, Bagher Larijani, et al. Giant prolactinoma: case report and review of literature. J Diabetes Metab Disor $2013 ; 12: 3$

6. Harzallah F, Harzallah L, Ben Brahim A, et al. Macroprolactinoma revealed by an exophtalmos. J Fr Ophtalmol. 2009;32(2):133.e1-133.e3.

7. Barkan AL, Chandler WF. Giant pituitary prolactinoma with falsely low serum prolactin: the pitfall of the "high-dose hook effect", case report. Neurosurgery. 1998;42(4):913-916.

8. Siddiqui A, Chew N, Miszkiel K. Unusual orbital invasion by a giant prolactinoma. Br J Radiol. 2008;81(971):e259-e262.

9. Berwaerts J, J Verhelst, R Abs, B Appel, et al. A giant prolactinoma presenting with unilateral exophthalmos: Effect of cabergoline and review of the literature. J Endocrinol Invest. 2000;23(6):393-398.

10. Cho EH, Lee SA, Chung JY, et al. Efficacy and safety of cabergoline as first line treatment for invasive giant prolactinoma. J Korean Med Sci. 2009;24(5):874-878.

11. Minniti G, Jaffrain Rea ML, Santoro A, et al. Giant prolactinomas presenting as skull base tumors. Surg Neurol. 2002;57(2):99-104.

12. Inagawa $H$, Ishizawa $K$, Mitsuhashi $T$, et al. Giant invasive pituitary adenoma extending into the sphenoid sinus and nasopharynx: report of a case with intraoperative cytologic diagnosis. Acta Cytol. $2005 ; 49(4): 452-456$

13. Care RH, Sunkaraneni VS, Theaker J, et al. Rapidly progressing giant invasive prolactinoma. J Laryngol Otol. 2012;126(8):840-843.

14. Shimon I, Benbassat C, Hadani M. Effectiveness of long-term cabergoline treatment for giant prolactinoma: study of 12 men. Eur J endocrinol. 2007;156(2):225-231

15. Acharya SV, Gopal RA, Menon PS, et al. Giant prolactinoma and effectiveness of medical management. Endocr Pract. 2010;16(1):42-46.

16. Cackett P, Eunson G, Bath L, et al. Proptosis as the presenting sign of giant prolactinoma in a prepubertal boy: successful resolution of hydrocephalus by use of medical therapy. Future Oncol. 2012;8(12):16211626 . 